ARTICLE OPEN

Check for updates

\title{
Laser-annealing Josephson junctions for yielding scaled-up superconducting quantum processors
}

\author{
Jared B. Hertzberg $\mathbb{D}^{1 凶}{ }^{凶}$, Eric J. Zhang ${ }^{1}$, Sami Rosenblatt $\mathbb{D}^{1}{ }^{1}$, Easwar Magesan ${ }^{1}$, John A. Smolin ${ }^{1}$, Jeng-Bang Yau ${ }^{1}$, \\ Vivekananda P. Adiga (iD), Martin Sandberg (iD ${ }^{1}$, Markus Brink (iD) ${ }^{1}$, Jerry M. Chow ${ }^{1}$ and Jason S. Orcutt (iD ${ }^{1}$
}

As superconducting quantum circuits scale to larger sizes, the problem of frequency crowding proves a formidable task. Here we present a solution for this problem in fixed-frequency qubit architectures. By systematically adjusting qubit frequencies postfabrication, we show a nearly tenfold improvement in the precision of setting qubit frequencies. To assess scalability, we identify the types of "frequency collisions" that will impair a transmon qubit and cross-resonance gate architecture. Using statistical modeling, we compute the probability of evading all such conditions, as a function of qubit frequency precision. We find that, without post-fabrication tuning, the probability of finding a workable lattice quickly approaches 0 . However, with the demonstrated precisions it is possible to find collision-free lattices with favorable yield. These techniques and models are currently employed in available quantum systems and will be indispensable as systems continue to scale to larger sizes.

npj Quantum Information (2021)7:129; https://doi.org/10.1038/s41534-021-00464-5

\section{INTRODUCTION}

Realizing robust large-scale quantum information processors is one of the foremost challenges in quantum science. Many practical applications have been proposed for robust quantum computers, including estimating the ground state energy of chemical compounds and implementing machine learning algorithms $s^{1-8}$. Quantum advantage relative to classical computers can be realized without full fault tolerance, but requires large quantum circuits that a classical computer cannot simulate ${ }^{9}$. Recent demonstrations have shown qubit circuits nearly at the threshold for demonstrating quantum advantage ${ }^{10}$. Much work remains in order to realize fault-tolerant quantum processors; however, scaleup of solid-state quantum circuits has shown consistent and ongoing progress ${ }^{11-20}$. As the qubit circuits are scaled up, they must maintain high one- and two-qubit gate fidelities, high qubit connectivity, and low cross-talk error, which can be measured in a holistic sense via the quantum volume of the circuit ${ }^{21,22}$. Lattices of fixed-frequency transmon qubits represent a promising architecture for building systems of larger sizes ${ }^{10}$. A growing number of systems at the 20-50-qubit scale are now available to users through cloud access. Fixed-frequency transmons are largely insensitive to charge or flux noise and have achieved coherence times of $100 \mu \mathrm{s}$ and growing. A variety of technical challenges confront further system scaling, including improving threedimensional circuit integration and fast readout. High on the list of such challenges is the issue of "frequency crowding."

The cross-resistance $(\mathrm{CR})$ gate, a hardware-efficient all-microwave gate ${ }^{23-26}$, is readily used to entangle fixed-frequency transmons with gate fidelities $>99 \%$, approaching the threshold for fault-tolerant $\operatorname{codes}^{27}$. To achieve these fidelities, the CR gate needs not only high coherence qubits but also a precise setting of the qubits' frequencies. The CR gate activates a $Z X$ interaction by driving one "control" qubit with a microwave pulse at the other "target" qubit's transition frequency. The magnitude of the $Z X$ as well as other Hamiltonian terms depends on the relative frequencies of the two qubits ${ }^{28,29}$. Diminished $Z X$ magnitude increases gate time, while other terms such as $Z Z$ add gate errors.
Neighboring qubits having the wrong detuning will exhibit a frequency collision in which the $Z X$ may be suppressed or other undesirable effects arise.

Maintaining high gate fidelities for all pairs in a lattice will require solving this frequency-crowding problem by precise setting of qubit frequencies to specified values, as characterized by a standard deviation $\sigma_{f}$. To achieve low $\sigma_{f}$, the tunnel-junction conductance must be controlled with high precision. Transmon frequency $f_{01}$ follows $h f_{01} \simeq \sqrt{8 E_{J} E_{C}}-E_{C}$, where Josephson energy $E_{J}=\frac{h l_{c}}{2 e}$ is many times greater than charging energy $E_{C}=\frac{e^{2} 30}{2 C}$. In typical transmons, a photolithographically defined capacitance $C$ has dimensions in the tens to hundreds of microns and varies little from qubit to qubit. The critical current $I_{c}$ is set by a tunnel barrier of area $\sim 100 \times 100 \mathrm{~nm}$ and thickness a few $\mathrm{nm}$ and is thus challenging to fabricate with precision better than a few percent ${ }^{31-35}$. However, tunnel barrier resistance $R_{\mathrm{n}}$ is readily measurable to precision better than $0.1 \%$ and relates to $I_{c}$ according to the Ambegaokar-Baratoff relation $I_{\mathrm{c}}=\frac{\pi \Delta}{2 e R_{\mathrm{n}}}$ (where $\Delta$ is the superconducting gap energy $)^{36}$. We expect imprecision in resistance $\sigma_{R}$ to produce a corresponding imprecision in frequency $\sigma_{f}=\frac{1}{2} \frac{\sigma_{R}}{\langle R\rangle} \cdot\langle f\rangle$, where $\langle f\rangle$ and $\langle R\rangle$ are the mean values of frequency and resistance, respectively. We can therefore measure $R_{\mathrm{n}}$ before a chip is cooled in order to assess qubit frequency imprecision. The best demonstrated precision in setting $R_{\mathrm{n}}$ at the time of fabrication is $2 \%^{34}$. A $2 \%$ variation in $R_{\mathrm{n}}$ indicates a fractional $\sigma_{f}$ of $1 \%$.

Careful design of lattices can enable error correction codes while at the same time minimizing the likelihood of "frequency collisions" and therefore the required $\sigma_{f}$ for fabrication yield ${ }^{37,38}$. Yet even the most robust designs require a fractional $\sigma_{f}$ of $0.25-0.5 \%$, which represents a factor of $2-4$ improvement over the best literature results. To overcome such limits will require rework of individual qubits' tunnel junctions after fabrication. Thermal anneal has been shown to increase tunnel resistance $R_{n}$, and laser heating has been demonstrated as a highly localized rework tool $^{39-44}$. However, the inherent variability of the anneal process itself must be overcome, and qubit frequency control utilizing

'IBM Quantum, IBM T.J. Watson Research Center, Yorktown Heights, NY, USA. ${ }^{凶}$ email: jbhertzberg@us.ibm.com 
such techniques at scale has never been presented in the literature.

In this paper, we introduce an adaptive post-fabrication trimming technique that we use to incrementally adjust $R_{\mathrm{n}}$ on a qubit-by-qubit basis, thereby overcoming inherent variability in both initial qubit fabrication and the laser anneal. We demonstrate this improvement in qubit frequency precision clearly in terms of narrowed frequency distributions. Crucially, we demonstrate qubit frequency imprecision $\sigma_{f}$ of the same magnitude as the imprecision of predicting $f_{01}$ from $R_{\mathrm{n}}$. To estimate the scalability of this technique for the fabrication of error-corrected lattices, we employ a statistical yield model based on $\sigma_{f}$ relative to specific collision bounds. This model predicts the severity of the frequency-crowding problem for different topologies and scales of error-corrected multi-qubit lattices as a function of code distance. The model demonstrates that, using conventional transmon fabrication, scaled-up qubit lattices will fail to evade frequency collisions. In contrast, our trimming technique achieves adequate $\sigma_{f}$ for scalable fabrication of distance-3 through distance-7 heavy-square and heavy-hexagon codes. In particular, this technique enables the high yield fabrication of the distance-3 and distance- 5 heavy-hexagon lattices currently deployed as IBM cloud connected systems ${ }^{22}$.

\section{RESULTS}

\section{Frequency precision $\sigma_{f}$ from transmon fabrication}

To assess the $\sigma_{f}$ resulting from qubit fabrication, we developed a test vehicle containing a large number of identically fabricated qubits (Fig. 1). We cooled the chip in a dilution refrigerator and used dispersive readout through half-wave microwave resonators to measure qubit frequencies ${ }^{45}$. We measured the frequencies of 31 qubits to a precision better than $100 \mathrm{kHz}$ using a Ramsey fringe method. The qubit frequencies had random variation $\sigma_{f}=$ $132.3 \mathrm{MHz}$ (Fig. 1) or $2.3 \%$ of the median frequency. After warming the qubits to room temperature, we measured their junction resistances. The standard deviation $\sigma_{R}$ was $365 \Omega, 4.6 \%$ of the median $R_{\mathrm{n}}$. Fractional $\sigma_{f}$ is exactly half of fractional $\sigma_{R}$, as expected from Transmon theory and the Ambegaokar-Baratoff relation. A plot of $R_{\mathrm{n}}$ against transmon frequency (Fig. 1) fits a power law of approximately $-\frac{1}{2}$ power, as expected from theory. To further assess the fidelity of the frequencies to this $f$-vs- $R_{\mathrm{n}}$ correlation, we show the residual scatter after subtracting the fit line. This appears in the inset in Fig. 1 and exhibits a standard deviation $14.5 \mathrm{MHz}$ or $0.25 \%$ of the qubit median frequency. Following transmon theory and the Ambegaokar-Baratoff relation, this residual scatter could indicate a qubit-to-qubit variation of up to $0.5 \%$ in superconducting gap $\Delta$ or qubit capacitance $C$. Small systematic errors in measuring $R_{n}$, for instance, due to substrate conductance, could also contribute. As we discuss below, future scaling of superconducting quantum logic circuits will require improvements in $\sigma_{f}$ and therefore better control of these parameters.

\section{Tuning using selective laser anneal}

To reduce $\sigma_{f}$, we developed a technique for selective laser anneal to shift tunnel resistance $R_{\mathrm{n}}$ by pre-calibrated increments (see "Methods" and Fig. 2). We demonstrate the achievable frequency control of this technique by shifting the 31 measured qubits into a two-frequency pattern. We employed an $R_{\mathrm{n}}$ vs $f$ correlation (Fig. 1) to designate the target resistances. We shifted 16 junctions to one $R_{\mathrm{n}}$ group and 15 to another $R_{\mathrm{n}}$ group. After tuning, the group of 16 junctions had median resistance $7.984 \mathrm{k} \Omega$ and the group of 15 had median resistance $8.798 \mathrm{k} \Omega$. The 31 junctions clustered around these medians with an overall precision of $\sigma_{R}=51 \Omega$, about $0.61 \%$. In a dilution refrigerator, we remeasured the frequencies of the qubits in the two groups. A change in fridge instrumentation degraded the readout signal-to-noise ratio of two of the qubits, so (a)

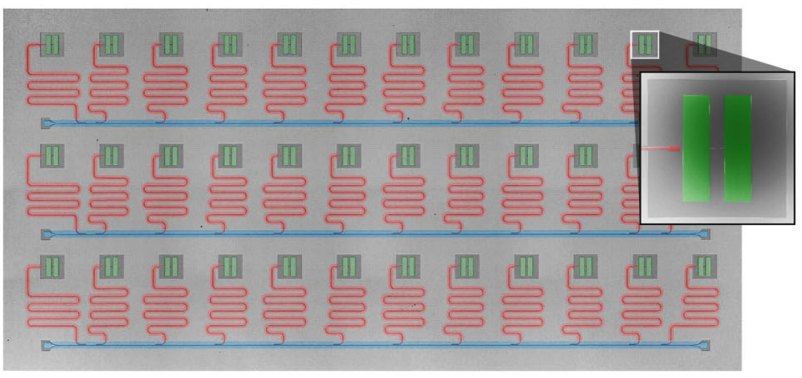

(b)
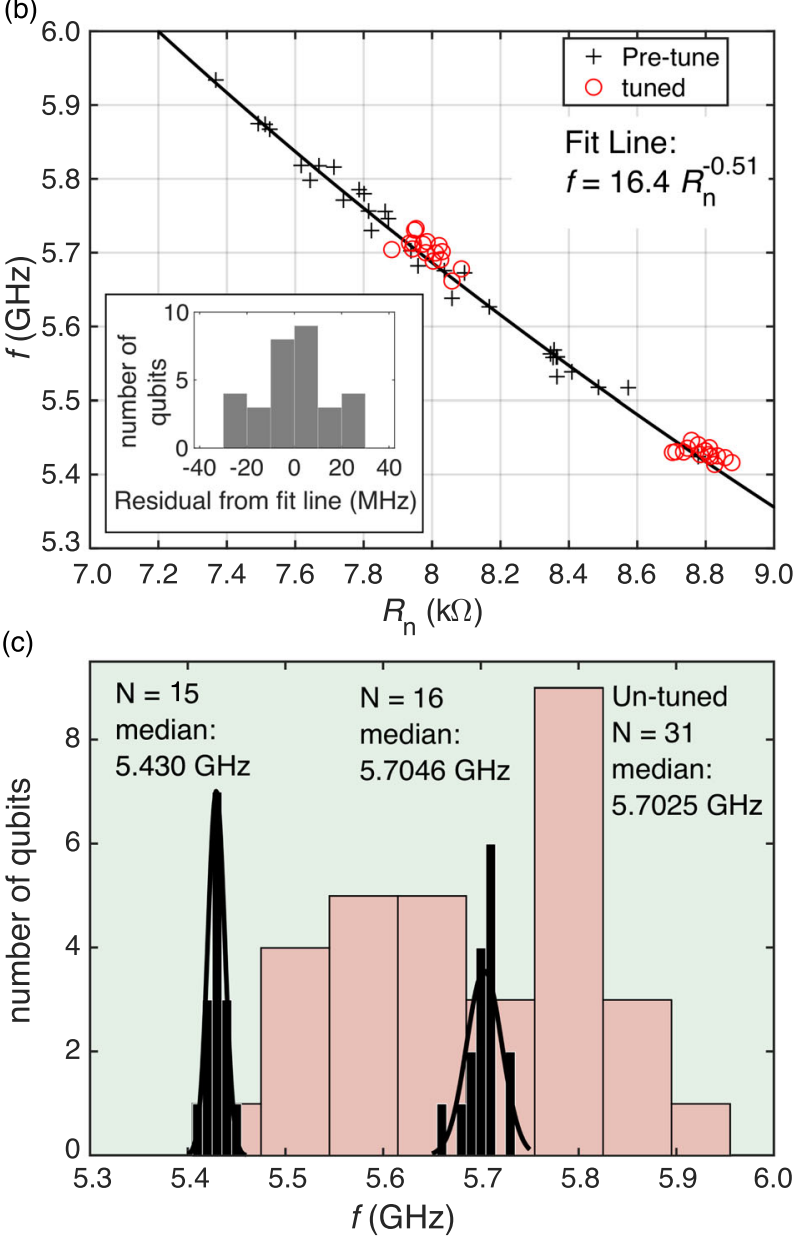

Fig. 1 Frequency precision $\sigma_{f}$ a False-colored image of test-vehicle chip. Thirty-six fixed-frequency transmon qubits, each including a $500 \times$ 320 micron planar capacitor (green) and a $~ 0.1 \times 0.1$ micron $\mathrm{Al} / \mathrm{AlO} \times / \mathrm{Al}$ tunnel junction, are prepared identically on a $20 \times 10 \mathrm{~mm}$ Si substrate. A half-wavelength coplanar waveguide resonator at each qubit (red) enables dispersive readout. Resonators are frequency-multiplexed in groups of 12 per feedline (blue). b Plot of transmon frequency vs $R_{n}$, and power-law fit of pre-tuned population. Inset histogram (10 MHz bins) shows residual scatter in frequency relative to fit line. c Distributions of qubit frequencies. Initial median was $5.7025 \mathrm{GHz}$ and initial spread was $\sigma_{f}=132.3 \mathrm{MHz}$ (red histogram, $70 \mathrm{MHz}$ bins). Using selective laser anneal (Fig. 2), we prepared these qubits into two distinct frequency populations with medians 5.430 and $5.7046 \mathrm{GHz}$ (black histograms, $10 \mathrm{MHz}$ bins). Each population is outlined by a Gaussian curve centered at its local median frequency. Combined spread is $\sigma_{f}=14.0 \mathrm{MHz}$.

that their remeasurement (using continuous-wave spectroscopy) had frequency precision of only $2 \mathrm{MHz}$. The others were remeasured in the same way as in the first cooldown. The resulting frequencies appear in Fig. 1. The two frequency groups 


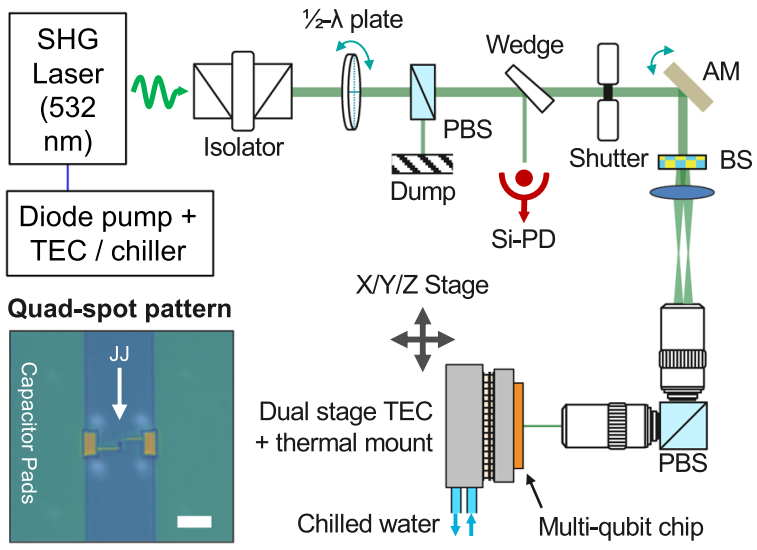

(a)

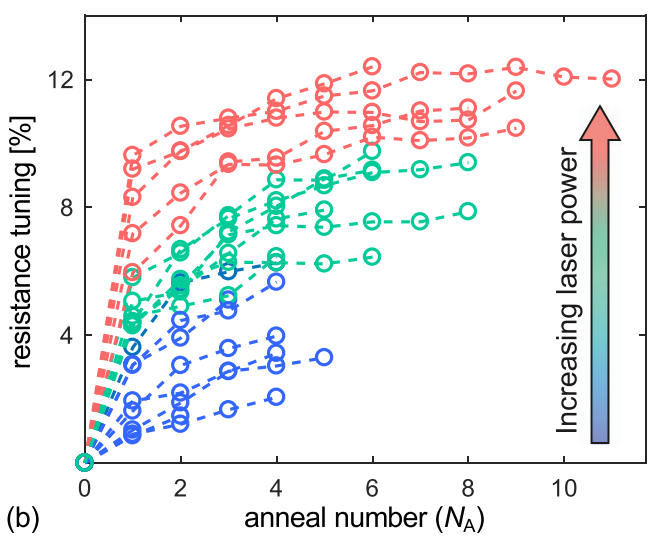

(b)

Fig. 2 "Laser annealing of stochastically impaired qubits" (LASIQ). a Schematic of the apparatus. A $532 \mathrm{~nm}$ (frequency doubled) diodepumped solid-state laser is used as the laser annealing source. Active power calibration is accomplished via a half-wave plate and PBS combination, with feedback from a Si-PD. A piezoelectric mirror mount actively aligns the beam to the junction center via image pattern recognition. The beam is shaped into a four spot pattern in an intermediate image plane by a diffractive beam splitter, to avoid direct illumination of the junction. The illumination pattern is condensed $4 \times$ using a dual-objective relay imaging set-up ${ }^{51,53}$. Inset with $10 \mu \mathrm{m}$ scale bar shows typical qubit junction and surrounding substrate heated by four Gaussian beam spots of diameter $\sim 4 \mu \mathrm{m}$. $\mathbf{b}$ Adaptive anneal progression toward $R_{\mathrm{n}}$ targets in 20 tunnel junctions. Greater fractional tuning requires greater anneal powers or durations. Incremental exposure (anneal number) permits tracking the resistance change so as to monotonically approach a target resistance and not overshoot it. In this demonstration, colors blue, green, and red correspond to laser powers $1.74,1.85$, and $1.96 \mathrm{~W}$, respectively. $R_{\mathrm{n}}$ shift was calibrated separately for each power. Single anneals lasted $\sim 0.3-8 \mathrm{~s}$. Total durations were $\sim 8-22 \mathrm{~s}$. SHG second harmonic generation, PBS polarizing beam splitter, Si-PD silicon photodiode, AM alignment mirror, BS beam splitter, TEC thermoelectric cooler.

are approximately normally distributed and have medians $f_{0,1}=$ $5.430 \mathrm{GHz}$ and $f_{0,2}=5.7046 \mathrm{GHz}$. Calculating $\sigma_{f}=\sqrt{\left\langle\left(f_{i}-f_{0, j}\right)^{2}\right\rangle}$, where $f_{0, j}$ represents $f_{0,1}$ or $f_{0,2}$ as appropriate for a given qubit $Q_{i}$ we assess the overall precision $\sigma_{f}=14.0 \mathrm{MHz}$. This imprecision is nearly identical to the residual scatter from the $f(R)$ fit line (Fig. 1), which guided the tuning, and the fractional precision $\sigma_{f} /\langle f\rangle=$ $0.25 \%$ is slightly better than half of the fractional precision in setting $R_{\mathrm{n}}$. Drift in $R_{\mathrm{n}}$ reported in the literature ${ }^{39}$ does not appear to be a limiting factor in this study. As we show in "Methods," the laser-anneal tuning technique is capable of precisions of $0.3 \%$ in $R_{\mathrm{n}}$. In future work, the imprecision of $14.5 \mathrm{MHz}$ in predicting $f$ from $R_{\mathrm{n}}$ could be made the limiting imprecision in $\sigma_{f}$.

\section{DISCUSSION}

Our post-fabrication trimming reduced $\sigma_{f}$ by $9.5 \times$ compared to initial fabrication. To assess whether this level of precision is sufficient to reliably prepare lattices of fixed-frequency transmons capable of error-correcting codes, we must quantify the frequency-crowding problem. Transmon qubits are weakly anharmonic and have decreasing transition energies at higher levels. Therefore, degeneracies among the $|0\rangle \rightarrow|1\rangle,|1\rangle \rightarrow|2\rangle$, and $|0\rangle \rightarrow|2\rangle$ transitions of nearby qubits can all contribute to frequency collisions. We must consider the relative frequencies of both nearest neighbors and next nearest neighbors in the lattice $28,46,47$. Figure 3 illustrates the relative positions of nearestneighbor and next-nearest-neighbor qubits in a section of lattice, and Table 1 lists the seven cases most likely to lead to gate errors $^{28}$. We can think of them qualitatively as follows: Type 1 causes hybridization of states in $Q_{j}$ and $Q_{k}$, while in type 2 the CR pulse excites $Q_{j}$ into the non-computational $|2\rangle$ state. Type 3 excites $Q_{k}$ to the $|2\rangle$ state but does not require a $\mathrm{CR}$ tone. In condition $4, Z X$ is weak, which implies long gate times and increased gate error ${ }^{28,29}$. In type 5, the CR gate addresses an additional neighboring qubit. In type 6 , when one qubit is the target of a CR gate, its next nearest neighbor leaks to the $|2\rangle$ state. In type 7, $Q_{j}$ acts as the control, $Q_{i}$ or $Q_{k}$ as the target, and the third qubit constitutes a "spectator." An excited-state spectator can emit a photon that combines with photons in the $C R$ pulse to excite $Q_{j}$ into the $|2\rangle$ state.

Around each of the frequency collisions described in Table 1, we can designate a window of undesired frequencies. This breaks the frequency space into allowed and forbidden regions. Type 4 listed in Table 1 defines forbidden zones where $Z X$ coupling is too low. For the other six conditions, we forbid regions where the frequency collision is the dominant source of gate error. Existing multi-qubit systems with CR gates typically exhibit two-qubit gate errors of $1-2 \%$ regardless of frequency ${ }^{15,46}$. Reference ${ }^{28}$ considers an effective Hamiltonian model for the CR gate, as a function of the relative frequency of control and target qubits. From this model, we estimate the frequency windows for nearest-neighbor collisions (Table 1, types 1-3). Within these windows, assuming typical gate parameters of $30-50 \mathrm{MHz}$ drive amplitude and 200-400 ns duration, we expect errors exceeding $\sim 1 \%$. Here we make an assumption that similar bounds apply to next-nearestneighbor interactions (types 5-7). Future lattice scaling will benefit from defining frequency collisions precisely to achieve specific quantum volume or error-correction thresholds. Such work can exploit numerical models $\mathrm{s}^{22,48}$ that find CR gate error as a function of frequency, coupling, and rate.

A useful lattice of qubits should enable high quantum volume and fault-tolerant operation while avoiding all of the frequency collisions and forbidden regions presented in Table 1. Both lattice layout and the pattern of qubit frequencies are relevant. We consider three types of lattices: square, "heavy square" and "heavy hexagon" (Fig. 3). Lattices comprise qubits and two-qubit connections, each qubit being linked to no more than four neighbors. In many practical implementations, these links comprise microwave-resonant buses. A square lattice facilitates "surface code" fault-tolerant $\operatorname{codes}^{49}$. Recent literature describes hybrids of the surface code with Bacon-Shor-type codes, which can be employed in heavy-hexagon and heavy-square lattices to achieve fault tolerance, albeit with lower error thresholds than the surface code $^{37}$. In addition to the data and ancilla qubit roles employed in the surface code, these hybrid codes assign a portion of the lattice as "flag" qubits. 


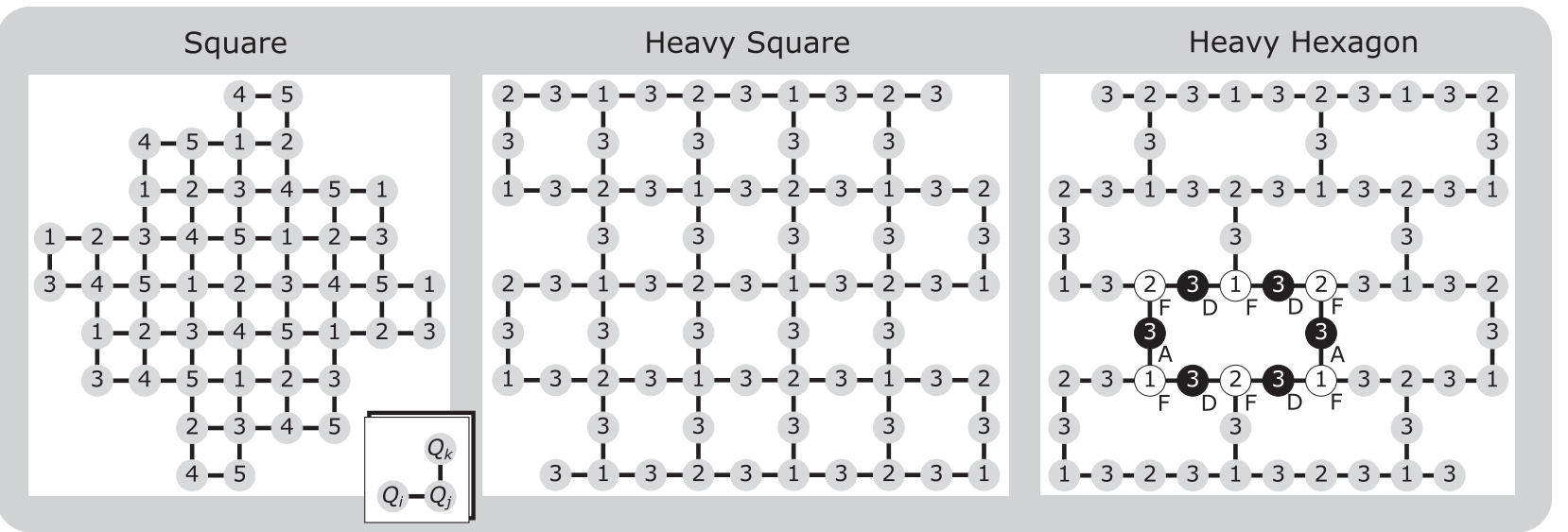

Fig. 3 Lattice and frequency pattern examples. Lattices are capable of $d=5$ codes. Patterns of qubit frequencies avoid all conditions in Table 1. Statistical model is applied to these examples and to equivalent lattices at $d=3$ and $d=7$ (see Supplementary Figs. 1-9). Square lattice includes 49 qubits in a 5-frequency pattern. Heavy-square lattice includes 73 qubits in 3-frequency pattern. Heavy-hexagon lattice includes 65 qubits in 3-frequency pattern. In a portion of the heavy-hexagon lattice, we indicate qubits' intended gate roles: control (black circles) or target (white circles), as well as code roles: data $(D)$, ancilla $(A)$, or flag $(F)^{37}$. Inset shows relative positions of qubits for collision definitions of Table 1. $Q_{j}$ is coupled to nearest-neighbors $Q_{i}$ and $Q_{k}$. Qubits $Q_{i}$ and $Q_{k}$ are next nearest neighbors.

Table 1. Seven most likely types of frequency collision.

\begin{tabular}{|c|c|c|c|}
\hline Type & Definition & Participants & Bounds \\
\hline 1 & $f_{j, 01}=f_{k, 01}$ & Nearest-neighbor qubits $Q_{j}, Q_{k}$ & $\pm 17 \mathrm{MHz}$ \\
\hline 2 & $f_{j, 02}=2 f_{k, 01}$ & Control qubit $Q_{j}$, target qubit $Q_{k}$ & $\pm 4 \mathrm{MHz}$ \\
\hline 3 & $f_{j, 01}=f_{k, 12}$ & Nearest-neighbor qubits $Q_{j}, Q_{k}$ & $\pm 30 \mathrm{MHz}$ \\
\hline 4 & $\begin{array}{l}f_{k, 01}<f_{j, 12} \text { or } f_{j, 01} \\
<f_{k, 01}\end{array}$ & Control qubit $Q_{j}$, target qubit $Q_{k}$ & - \\
\hline 5 & $f_{i, 01}=f_{k, 01}$ & $\begin{array}{l}Q_{j} \text { is control to } Q_{i} \text { and/or } Q_{k} \text { and is } \\
\text { nearest neighbor to both }\end{array}$ & $\pm 17 \mathrm{MHz}$ \\
\hline 6 & $\begin{array}{l}f_{i, 01}=f_{k, 12} \text { or } f_{i, 12} \\
=f_{k, 01}\end{array}$ & $\begin{array}{l}Q_{j} \text { is control to } Q_{i} \text { and/or } Q_{k} \text { and is } \\
\text { nearest neighbor to both }\end{array}$ & $\pm 25 \mathrm{MHz}$ \\
\hline 7 & $f_{j, 02}=f_{k, 01}+f_{i, 01}$ & $\begin{array}{l}Q_{j} \text { is control to } Q_{i} \text { and/or } Q_{k} \text { and is } \\
\text { nearest neighbor to both }\end{array}$ & $\pm 17 \mathrm{MHz}$ \\
\hline
\end{tabular}

For bus-coupled transmon qubits employing cross-resonance gates and having anharmonicity $\sim-330 \mathrm{MHz}$. Relative qubit positions illustrated in inset of Fig. 3. Bounds for types 1-3 are estimated from model results ${ }^{28}$ as the frequency region around each degeneracy in which gate errors due to frequency collisions exceed $\sim 1 \%$. For instance, nearest-neighbor qubits $Q_{j}$ and $Q_{k}$ exhibit a type 1 collision if their frequencies obey $f_{k, 01}-17 \mathrm{MHz}<$ $f_{j, 01}<f_{k, 01}+17 \mathrm{MHz}$. Bounds for types $5-7$ are based on those in types 1 and 3 .

In the square lattice, every qubit in the bulk of the lattice lies on a degree-four vertex, while some at edges have degree two or degree three. If we populate the square lattice with five distinct frequencies of qubits, $f_{5}>f_{4}>f_{3}>f_{2}>f_{1}$, with appropriate spacing between the frequencies, we can avoid all the forbidden regions of Table $1^{13}$. In Fig. 3, we illustrate this pattern for a square lattice capable of a distance-5 $(d=5)$ rotated surface code. Condition 4 of Table 1 requires $f_{\text {control }}>f_{\text {target, }}$ so the pattern also fixes the direction of CNOT gate for each pair.

In contrast to the square lattice, the heavy-square lattice includes both degree-two and degree-four vertices in the bulk. Degree-one, degree-two, or degree-three vertices appear at the edges. We take advantage of this pattern to make all the degreetwo vertices control qubits, using a three-frequency pattern $f_{3}>f_{2}$ $>f_{1}$. Since every control qubit (frequency $f_{3}$ ) is linked to at most two target qubits, we need only two properly chosen target-qubit frequencies $\left(f_{1}\right.$ and $\left.f_{2}\right)$ to satisfy conditions $5-7$ of Table 1 , as shown in Fig. 3. A third type of lattice, the heavy hexagon, uses a similar scheme. Here the bulk of the lattice includes degree-three and degree-two vertices. Additional degree-two and degree-one vertices lie at the edges. In this lattice, all of the frequency collisions and forbidden regions can be satisfied using only three frequencies $f_{3}>f_{2}>f_{1}$, with all control qubits residing on degreetwo vertices with frequency $f_{3}$.

We use a Monte Carlo model to quantify the frequency crowding in each lattice type. We sample the qubits at random frequencies drawn from normal distributions characterized by $\sigma_{f}$ and count the collisions defined in Table 1 (see "Methods" and Fig. 6). In Fig. 4, we show the mean number of frequency collisions predicted by the Monte Carlo model for each lattice type and frequency pattern, as a function of $\sigma_{f}$. As $\sigma_{f} \rightarrow 0$, the lattice approaches the ideal patterns of Fig. 3 and has zero frequency collisions. As $\sigma_{f}$ increases, the number of frequency collisions rises steadily. As $\sigma_{f} \rightarrow f_{01}-f_{12}$, the different conditions appearing in Table 1 all become likely, and a limiting number of frequency collisions is reached. Yield follows the inverse trend, as seen in Fig. 4. As $\sigma_{f}$ increases, the likelihood of finding a "collision-free" chip falls off sharply. While the step sizes between frequency setpoints $f_{1}$ to $f_{5}$ are important, absolute values of setpoints are not. Setting $f_{1}=5.0, f_{2}=5.07$, and $f_{3}=5.14 \mathrm{GHz}$ works as well as $f_{1}=5.05, f_{2}=$ 5.12 , and $f_{3}=5.19 \mathrm{GHz}$.

The yield and mean collision number are a function of the several different collision types and bounds, so they are not readily susceptible to an analytic formulation. However, we can propose a simplified model for yield: in order for a lattice to be collision-free, every qubit in the lattice must fall within some frequency "window" $\pm \Delta f$ relative to its setpoint. Presuming the qubit frequencies are normally distributed, the probability of this occurring goes as the cumulative distribution function, raised to the power $N$, where $N$ is the number of qubits: $\left[\int_{-\infty}^{\left(\Delta f / \sigma_{f}\right)} e^{-\frac{1}{2} x^{2}} \mathrm{~d} x\right]^{N}$. In the yield plot in Fig. 4, we fit this expression to find $\Delta f$ for each lattice.

These model results allow us to predict how different lattice types and frequency patterns will respond to fabrication imprecision. As shown in Fig. 4, if imprecision $\sigma_{f}$ is $>30 \mathrm{MHz}$, any $d=5$ lattice will exhibit $>10$ frequency collisions of one or another of the types listed in Table 1, causing the affected gates to have error rates above $\sim 1 \%$. However, if $\sigma_{f}=10 \mathrm{MHz}$ then on average the $d$ $=5$ square lattice will exhibit 5 frequency collisions, while the heavy-square and heavy-hexagon lattices will exhibit 0.1 frequency collision. Considered in terms of yield, we see from Fig. 4 

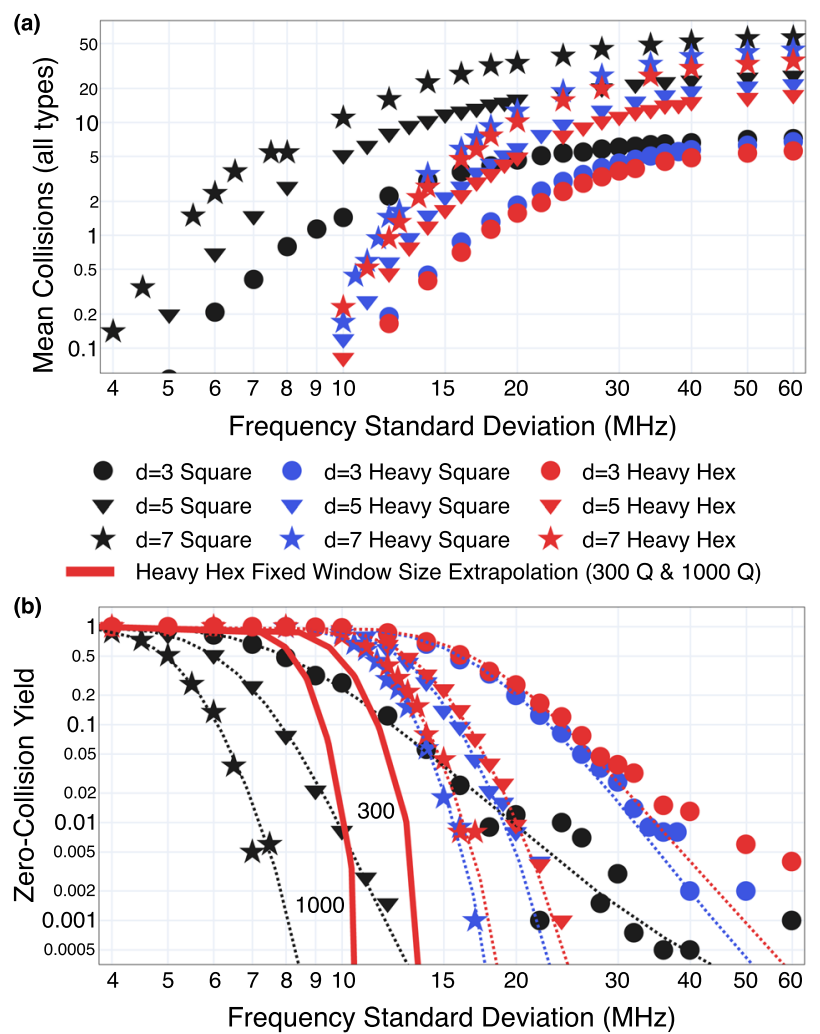

Fig. 4 Frequency-crowding trends as a function of $\sigma_{f}$. Results of Monte Carlo simulation (see "Methods"). Simulation was applied to the lattices and frequency patterns shown in Fig. 3, capable of distance- 5 codes, as well as to $d=3$ and $d=7$ scale lattices of square, heavy-square, or heavy-hexagon type (See Supplementary Figs. 1-9 for lattice layouts and Table 2 for numbers of qubits.). a Average number of collisions. b Fraction of cases having zero frequency collisions. Color-coded dotted lines are fits of each lattice yield to expression $\left[\int_{-\infty}^{\left(\Delta f / \sigma_{f}\right)} e^{-\frac{1}{2} x^{2}} d x\right]^{N}$, where $N$ is the number of qubits and $\pm \Delta f$ defines an allowable window around frequency set points (see Table 2). Using this expression, two solid red lines predict yield for the heavy-hexagon lattice type at 300 and 1000 qubits, using $\Delta f=27.99$ and $26.32 \mathrm{MHz}$, respectively. See Fig. 5 for estimation of $\Delta f$ as a function of qubit number.

that if $\sigma_{f}=10 \mathrm{MHz}$, then for a $d=5$ device, a square lattice with 5 -frequency pattern has a $0.8 \%$ likelihood to be collision free, whereas a heavy-square lattice with 3-frequency pattern has $90 \%$ likelihood and heavy hexagon with 3-frequency pattern has $92 \%$ likelihood. Alternatively we can ask, how well do we have to control $\sigma_{f}$ ? If we seek a $10 \%$ yield, then Fig. 4 indicates that, for a $d=5$ device, a square lattice with 5 -frequency pattern requires $\sigma_{f}$ $<8 \mathrm{MHz}$, whereas a heavy-square lattice with 3-frequency pattern requires $\sigma_{f}=16 \mathrm{MHz}$ and heavy hexagon with 3-frequency pattern requires $\sigma_{f}=17 \mathrm{MHz}$. Although the square lattice requires $10-20 \%$ fewer qubits than the other types at each distance $d$, it requires far better frequency precision.

The as-fabricated $\sigma_{f}$ seen in Fig. 1 is $132.3 \mathrm{MHz}$ (see "Results"). The Monte Carlo modeling finds that for a heavy-hexagon lattice at $d=3$ scale this $\sigma_{f}$ can enable $0.1 \%$ yield of collision-free chips. Other lattice types and larger scales will all have yield $\ll 0.1 \%$. The re-tuned $\sigma_{f}=14.0 \mathrm{MHz}$ demonstrated in Fig. 1 will improve the yield in all types of lattice. Predictions of the Monte Carlo model for $\sigma_{f}=14.0 \mathrm{MHz}$ appear in Table 2. At $d=5$ scale, the heavyhexagon and heavy-square lattices and 3-frequency patterns should be collision-free nearly one-third of the time, while at $d=7$ scale the yield is about four times smaller, still reasonable for prototype systems.

As seen from the Monte Carlo analysis, the laser-anneal rework method can scale to the $>100$ qubit size, enabling a well-chosen lattice and frequency pattern to implement $d=7$ error-correction codes free of frequency crowding. To examine needs for the next generation of chips up to the 1000-qubit level, we can coarsely estimate requirements by extrapolating the fixed window model for the heavy-hexagon lattice as shown in Fig. 5. While the $\sigma_{f}=14.0 \mathrm{MHz}$ demonstrated here enables practical yield up to the 100-200 qubit scale, it is clear that roughly a factor of two further improvement is needed to scale toward 1000 qubits. Since this precision is also better than the resistance-to-frequency prediction precision shown in this work, development of further refinements in tuning and frequency prediction approaches will be necessary as the scale of fixedfrequency transmon circuits surpass the 100 qubit milestone.

\section{METHODS}

\section{Chip fabrication}

A chip of the kind used to determine $\sigma_{f}$ and to test our laser-anneal rework process appears in Fig. 1. All microwave elements comprise $\mathrm{Nb}$ films $\sim 200 \mathrm{~nm}$ thick on a silicon substrate. Each qubit is coupled to a readout resonator but is not directly coupled to any nearby qubits. All transmon capacitors are identical. Junctions are fabricated using identical electronbeam lithographic patterns and deposited simultaneously using doubleangle deposition and oxidation ${ }^{50}$. The individual qubit design is similar to that used in ref. ${ }^{27}$ with anharmonicity $f_{12}-f_{01} \simeq-330 \mathrm{MHz}$. Junctions have linear dimension $\sim 100 \mathrm{~nm}$ and are designed for $I_{\mathrm{c}}$ of $\sim 30 \mathrm{nA}$. During packaging, we accidentally damaged 3 of the 36 qubits and found these to be non-functional when cooled in a dilution refrigerator. We left 2 of the remaining 33 qubits un-tuned as experimental controls, so that our tuning demonstration includes 31 qubits.

\section{Tuning using selective laser anneal}

We have built an integrated junction rework system that can measure and modify the junction resistance. Figure 2 shows a schematic of our laser annealing system, which we call Laser Annealing of Stochastically Impaired Qubits (LASIQ). The laser output is generated by a diode-pumped solidstate laser, frequency doubled to $532 \mathrm{~nm}$. Active power control of the anneal beam from approximately 1.7 to $2 \mathrm{~W}$ is performed using a piezorotary mounted waveplate and polarizing beam splitter, which is adaptively adjusted based on a pick-off beam measured on a downstream silicon photodiode. A precision-timed shutter exposes the device for $0.3-10 \mathrm{~s}$, and beam alignment is performed using a mechanical mirror mount, which directs the beam via pattern recognition to the transmon junction center. To achieve a more consistent anneal, the beam is shaped into a four-spot pattern, which avoids directly illuminating the junction but uniformly heats the surrounding substrate ${ }^{51}$.

By careful control of laser power and pulse duration, we use this system to adjust $R_{\mathrm{n}}$. This process overcomes the imprecision due to transmon fabrication, with a residual imprecision $\sigma_{f}$ due to the rework process. To develop the process, we prepared a set of 126 junctions identical to qubit junctions and measured their response to a range of laser powers and exposure times. We recorded $R_{\mathrm{n}}$ shifts up to $15 \%$ relative to initial $R_{\mathrm{n}}$ for total anneal durations varying from 2 to $80 \mathrm{~s}$ and laser powers varying from 1.6 to $2 \mathrm{~W}$. Response to laser power in particular was highly nonlinear. Based on these empirical calibrations of $R_{\mathrm{n}}$ shift to power and exposure, we established a qubit tuning process: We first measure the transmon junction's $R_{\mathrm{n}}$ using four-point probing of the transmon capacitor pads at $25^{\circ} \mathrm{C}$. Using a $f\left(R_{n}\right)$ prediction based on a previously determined correlation curve (Fig. 1), we assign the junction a target resistance corresponding to the target frequency in a multiqubit chip lattice. Because the anneal can shift $R_{\mathrm{n}}$ in only one direction, the target must be higher than the initial $R_{\mathrm{n}}$. We anneal the qubit junction using laser power and duration chosen from our calibration set, then remeasure its $R_{n}$. By remeasuring after each anneal, we can adjust for random variation in the amount of $R_{\mathrm{n}}$ shift. A junction requiring large shifts in $R_{\mathrm{n}}$ may require repeated anneals to reach its target, as shown in Fig. 2. The control algorithm increases the resistance until the measured value is within $0.3 \%$ of the target value. In a separate trial of tuning precision, $>300$ junctions were tuned to target $R_{\mathrm{n}} \mathrm{s}$ ranging from 0.4 to $14.5 \%$ above their initial 
J.B. Hertzberg et al.

Table 2. Monte Carlo model predictions of the mean number of collisions and yield of collision-free chips.

\begin{tabular}{|c|c|c|c|c|c|c|c|}
\hline \multirow{2}{*}{$\begin{array}{l}\text { Lattice and frequency } \\
\text { pattern }\end{array}$} & \multirow{2}{*}{$\begin{array}{l}\text { Code } \\
\text { distance }\end{array}$} & \multirow[t]{2}{*}{ Qubits } & \multicolumn{2}{|l|}{$\sigma_{f}=132.3 \mathrm{MHz}$} & \multicolumn{2}{|l|}{$\sigma_{f}=14 \mathrm{MHz}$} & \multirow[t]{2}{*}{$\Delta f$ Window $(\mathrm{MHz})$} \\
\hline & & & $\begin{array}{l}\text { Mean number of } \\
\text { collisions }\end{array}$ & $\begin{array}{l}\text { Yield of collision- } \\
\text { free devices }\end{array}$ & $\begin{array}{l}\text { Mean number of } \\
\text { collisions }\end{array}$ & $\begin{array}{l}\text { Yield of collision- } \\
\text { free devices }\end{array}$ & \\
\hline \multirow[t]{3}{*}{ Square, 5-frequency } & $d=3$ & 17 & 9 & $\ll 0.1 \%$ & 3 & $6 \%$ & 13.96 \\
\hline & $d=5$ & 49 & 35 & $\ll 0.1 \%$ & 10 & $<0.1 \%$ & 13.23 \\
\hline & $d=7$ & 97 & 78 & $\ll 0.1 \%$ & 23 & $\ll 0.1 \%$ & 12.12 \\
\hline $\begin{array}{l}\text { Heavy-square, } \\
\text { 3-frequency }\end{array}$ & $d=7$ & 145 & 70 & $\ll 0.1 \%$ & 3.5 & $6 \%$ & 29.06 \\
\hline \multirow{3}{*}{$\begin{array}{l}\text { Heavy-hexagon, } \\
\text { 3-frequency }\end{array}$} & $d=3$ & 23 & 8 & $0.1 \%$ & 0.4 & $70 \%$ & 31.61 \\
\hline & $d=5$ & 65 & 25 & $\ll 0.1 \%$ & 1.2 & $33 \%$ & 29.91 \\
\hline & $d=7$ & 127 & 51 & $\ll 0.1 \%$ & 2.7 & $8 \%$ & 29.29 \\
\hline
\end{tabular}

Full results of Monte Carlo model of frequency crowding appear in Fig. 4. Here we show values for as-fabricated precision, $\sigma_{f}=132.3 \mathrm{MHz}$, and for demonstrated frequency precision of the laser-anneal method, $\sigma_{f}=14 \mathrm{MHz}$ (Fig. 1), in different lattices and frequency patterns (Fig. 3 and Supplementary Figs. 1-9). The $\Delta f$ values correspond to the fit lines in Fig. 4: in order for the device to be collision-free, every qubit in the lattice must fall within \pm this window, relative to its set point.

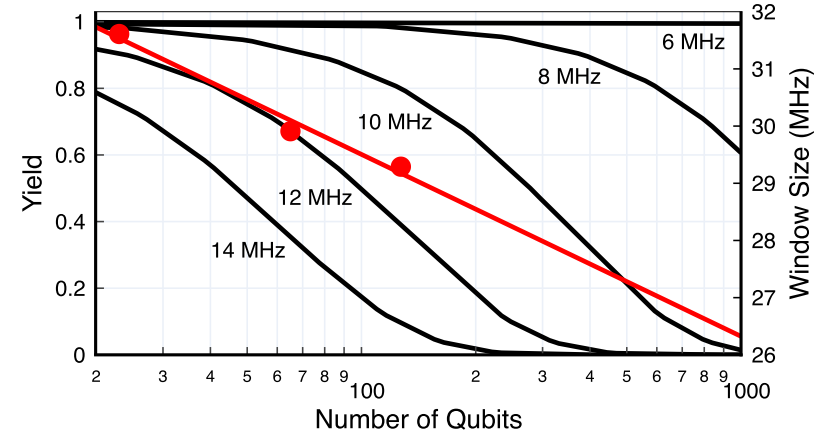

Fig. 5 Yield scaling for heavy-hexagon lattice. Fixed window size model. (Back curves, left axis) Model yields for $\sigma_{f}$ ranging from the $14 \mathrm{MHz}$ demonstrated in this work to $6 \mathrm{MHz}$ that would optimize future scaling yield beyond the $1000 \mathrm{Q}$ level. (Red points and curve, right axis) Fixed window size fits to the $d=3, d=5$, and $d=7$ heavyhexagon lattice, as well as a fit of these values to expression $A+B \cdot \log (N)$, extrapolated from 20 to 1000 qubits. This trend illustrates varying frequency-crowding constraints as a function of lattice size.

values and landed successfully within this $0.3 \%$ margin. We observed this precision to be independent of the target $R_{\mathrm{n}}$. We expect $0.3 \%$ imprecision in $R_{\mathrm{n}}$ to introduce $0.15 \%$ imprecision in transmon frequency.

\section{Monte Carlo frequency-crowding model}

Using a Monte Carlo model, we can estimate the incidence of frequency collisions in a lattice as a function of $\sigma_{f}$. We assume that imperfect frequency setting will distribute qubit frequencies normally around their design frequencies with standard deviation $\sigma_{f}$. For lattices of the type shown in Fig. 3 , we designate 3-5 frequencies $f_{1}, f_{2}, f_{3}, f_{4}, f_{5}$ spaced at regular intervals in the pattern shown. We set $f_{1}=5 \mathrm{GHz}$, similar to realworld transmons ${ }^{22,52}$. We sample the qubit frequencies randomly around these values and count the collisions throughout the lattice, as listed in Table 1. To avoid unnecessary counting of type 4, we designate the higherfrequency qubit of every pair to be the control for that gate pair. This process is illustrated in Fig. 6. We repeat the frequency assignment and counting to build statistics for a given lattice and frequency pattern. We then repeat the model for a range of $\sigma_{f}$ values from 0 to $150 \mathrm{MHz}$. We repeat the entire process over a range of frequency spacings to find the spacing that minimizes frequency collisions at each value of $\sigma_{f}$. As a function of $\sigma_{f}$, we can then extract (1) the mean number of total collisions
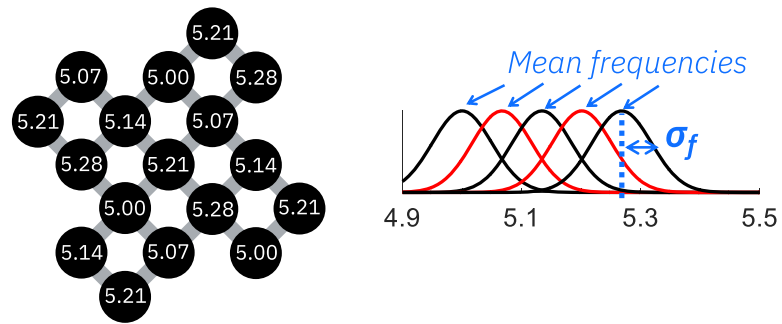

Fig. 6 Frequency collision statistical model. Left: Square lattice, $d=3$ with 5 -frequency pattern of Fig. $3: f_{1}=5.00, f_{2}=5.07, f_{3}=5.14$, $f_{4}=5.21, f_{5}=5.28 \mathrm{GHz}$. To model the lattice statistically, treat the frequencies $f_{1}$ to $f_{5}$ as means of distributions. Right: Mean frequencies and normal distributions characterized by $\sigma_{f}$. For each position in the lattice, sample from the local distribution. Choose random frequencies in this fashion, count collisions as described in Table 1, and repeat to gather statistical sample. Process is repeated for differing spacings between mean frequencies $f_{1}$ to $f_{5}$ and for different distribution widths $\sigma_{f}$.

in the lattice and (2) the fraction of repetitions that result in zero collisions (yield). Our simulations used 1000 repetitions except to find yield $<1 \%$ in $d=5$ lattices and $<0.2 \%$ in $d=3$ lattices, which used 4000 repetitions, and in $d=7$ lattices to find mean collisions for $\sigma_{f}<16 \mathrm{MHz}$ or yield $>50 \%(100$ repetitions) or to find mean collisions for $\sigma_{f}>16 \mathrm{MHz}$ (40 repetitions).

\section{DATA AVAILABILITY}

The experimental data presented in this manuscript are available from the corresponding author upon reasonable request.

Received: 7 September 2020; Accepted: 19 April 2021; Published online: 19 August 2021

\section{REFERENCES}

1. Kandala, A. et al. Hardware-efficient variational quantum eigensolver for small molecules and quantum magnets. Nature 549, 242-246 (2017).

2. Havlíček, V. et al. Supervised learning with quantum-enhanced feature spaces. Nature 567, 209-212 (2019).

3. Hempel, C. et al. Quantum chemistry calculations on a trapped-ion quantum simulator. Phys. Rev. X 8, 031022 (2018). 
4. Colless, J. et al. Computation of molecular spectra on a quantum processor with an error-resilient algorithm. Phys. Rev. X 8, 011021 (2018).

5. Nam, Y. et al. Ground-state energy estimation of the water molecule on a trapped-ion quantum computer. npj Quantum Inf. 6, 1-6 (2020).

6. Arute, F. et al. Hartree-Fock on a superconducting qubit quantum computer. Science 369, 1084-1089 (2020).

7. Argüello-Luengo, J., González-Tudela, A., Shi, T., Zoller, P. \& Cirac, J. I. Analogue quantum chemistry simulation. Nature 574, 215-218 (2019).

8. McArdle, S., Endo, S., Aspuru-Guzik, A., Benjamin, S. C. \& Yuan, X. Quantum computational chemistry. Rev. Mod. Phys. 92, 015003 (2020).

9. Kandala, A. et al. Error mitigation extends the computational reach of a noisy quantum processor. Nature 567, 491-495 (2019).

10. Córcoles, A. D. et al. Challenges and opportunities of near-term quantum computing systems. Proc. IEEE 1-15 https://doi.org/10.1109/JPROC.2019.2954005 (2019).

11. Chow, J. M. et al. Implementing a strand of a scalable fault-tolerant quantum computing fabric. Nat. Commun. 5, 4015 (2014).

12. Córcoles, A. et al. Demonstration of a quantum error detection code using a square lattice of four superconducting qubits. Nat. Commun. 6, 6979 (2015).

13. Gambetta, J. M., Chow, J. M. \& Steffen, M. Building logical qubits in a superconducting quantum computing system. npj Quantum Inf. 3, 2 (2017).

14. Takita, M. et al. Demonstration of weight-four parity measurements in the surface code architecture. Phys. Rev. Lett. 117, 210505 (2016).

15. Takita, M., Cross, A. W., Córcoles, A. D., Chow, J. M. \& Gambetta, J. M. Experimental demonstration of fault-tolerant state preparation with superconducting qubits. Phys. Rev. Lett. 119, 180501 (2017).

16. Kelly, J. et al. State preservation by repetitive error detection in a superconducting quantum circuit. Nature 519, 66-69 (2015).

17. Risté, D. et al. Detecting bit-flip errors in a logical qubit using stabilizer measurements. Nat. Commun. 6, 6983 (2015).

18. Ofek, N. et al. Extending the lifetime of a quantum bit with error correction in superconducting circuits. Nature 536, 441-445 (2016).

19. Arute, F. et al. Quantum supremacy using a programmable superconducting processor. Nature 574, 505-510 (2019).

20. Kjaergaard, M. et al. Superconducting qubits: current state of play. Annu. Rev. Condens. Matter Phys. 11, 369-395 (2020).

21. Cross, A. W., Bishop, L. S., Sheldon, S., Nation, P. D. \& Gambetta, J. M. Validating quantum computers using randomized model circuits. Phys. Rev. A 100, 032328 (2019).

22. Jurcevic, P. et al. Demonstration of quantum volume 64 on a superconducting quantum computing system. Preprint at https://doi.org/10.1088/2058-9565/ abe519 (2020).

23. Chow, J. M. et al. Simple all-microwave entangling gate for fixed-frequency superconducting qubits. Phys. Rev. Lett. 107, 080502 (2011)

24. Chow, J. M. et al. Universal quantum gate set approaching fault-tolerant thresholds with superconducting qubits. Phys. Rev. Lett. 109, 060501 (2012).

25. de Groot, P. C. et al. Selective darkening of degenerate transitions for implementing quantum controlled-NOT gates. N. J. Phys. 14, 073038 (2012).

26. Rigetti, C. \& Devoret, M. Fully microwave-tunable universal gates in superconducting qubits with linear couplings and fixed transition frequencies. Phys. Rev. B 81, 134507 (2010).

27. Sheldon, S., Magesan, E., Chow, J. M. \& Gambetta, J. M. Procedure for systematically tuning up crosstalk in the cross resonance gate. Phys. Rev. A 93, 060302 (2016).

28. Magesan, E. \& Gambetta, J. M. Effective Hamiltonian models of the crossresonance gate. Phys. Rev. A 101, 052308 (2020).

29. Ware, $M$. et al. Cross-resonance interactions between superconducting qubits with variable detuning. Preprint at http://arxiv.org/abs/1905.11480v1 (2019).

30. Koch, J. et al. Charge-insensitive qubit design derived from the Cooper pair box. Phys. Rev. A 76, 042319 (2007).

31. Potts, A., Parker, G., Baumberg, J. \& de Groot, P. CMOS compatible fabrication methods for submicron Josephson junction qubits. IEE Proc. Sci. Meas. Technol. 148, 225-228 (2001).

32. Wu, X. et al. Overlap junctions for high coherence superconducting qubits. Appl. Phys. Lett. 111, 032602 (2017).

33. Costache, M. V., Bridoux, G., Neumann, I. \& Valenzuela, S. O. Lateral metallic devices made by a multiangle shadow evaporation technique. J. Vac. Sci. Technol. B 30, 04E105 (2012).

34. Kreikebaum, J. M., O'Brien, K. P., Morvan, A. \& Siddiqi, I. Improving wafer-scale Josephson junction resistance variation in superconducting quantum coherent circuits. Supercond. Sci. Technol. 33, 06LT02 (2020).

35. Foroozani, N. et al. Development of transmon qubits solely from optical lithography on 300 mm wafers. Quantum Sci. Technol. 4, 025012 (2019).

36. Ambegaokar, V. \& Baratoff, A. Tunneling between superconductors. Phys. Rev. Lett. 10, 486-489 (1963).
37. Chamberland, C., Zhu, G., Yoder, T. J., Hertzberg, J. B. \& Cross, A. W. Topological and subsystem codes on low-degree graphs with flag qubits. Phys. Rev. $X 10$ 011022 (2020).

38. Chow, J. M., Magesan, E., Steffen, M., Gambetta, J. M. \& Takita, M. Reducing qubit frequency collisions through lattice design. US patent $(10,622,536)$ US20200161529A1 (2020).

39. Koppinen, P. J., Väistö, L. M. \& Maasilta, I. J. Complete stabilization and improvement of the characteristics of tunnel junctions by thermal annealing. Appl. Phys. Lett. 90, 053503 (2007).

40. Granata, C. et al. Trimming of critical current in niobium Josephson devices by laser annealing. J. Phys. Conf. Ser. 97, 012110 (2008).

41. Muthusubramanian, N. et al. Local trimming of transmon qubit frequency by laser annealing of Josephson junctions. In American Physical Society March Meeting B29.015 (American Physical Society, 2019).

42. Oliva, A. \& Monaco, R. Annealing properties of high quality $\mathrm{Nb} / \mathrm{Al}-\mathrm{AlO}{ }_{x} / \mathrm{Nb}$ tunnel junctions. IEEE Trans. Appl. Supercond. 4, 25-32 (1994).

43. Lehnert, T., Billon, D., Grassl, C. \& Gundlach, K. H. Thermal annealing properties of $\mathrm{Nb}-\mathrm{Al} / \mathrm{AlO}_{x}-\mathrm{Nb}$ tunnel junctions. J. Appl. Phys. 72, 3165-3168 (1992).

44. Rosenblatt, S., Orcutt, J. S. \& Chow, J. M. Laser annealing qubits for optimized frequency allocation. US patent 10340438B2 (2019).

45. Blais, A., Huang, R.-S., Wallraff, A., Girvin, S. M. \& Schoelkopf, R. J. Cavity quantum electrodynamics for superconducting electrical circuits: an architecture for quantum computation. Phys. Rev. A 69, 062320 (2004).

46. McKay, D. C., Sheldon, S., Smolin, J. A., Chow, J. M. \& Gambetta, J. M. Three-qubit randomized benchmarking. Phys. Rev. Lett. 122, 200502 (2019).

47. Malekakhlagh, M., Magesan, E. \& McKay, D. C. First-principles analysis of crossresonance gate operation. Phys. Rev. A 102, 042605 (2020).

48. Sundaresan, N. et al. Reducing unitary and spectator errors in cross resonance with optimized rotary echoes. PRX Quantum 1, 020318 (2020).

49. Fowler, A. G., Mariantoni, M., Martinis, J. M. \& Cleland, A. N. Surface codes: towards practical large-scale quantum computation. Phys. Rev. A 86, 032324 (2012).

50. Dolan, G. J. Offset masks for lift-off photoprocessing. Appl. Phys. Lett. 31, 337-339 (1977).

51. Rosenblatt, S. \& Orcutt, J. S. Laser annealing of qubits with structured illumination. US patent 10170681B1 (2019).

52. Sheldon, $\mathrm{S}$. et al. Characterizing errors on qubit operations via iterative randomized benchmarking. Phys. Rev. A 93, 012301 (2016).

53. Orcutt, J. S. Laser annealing of qubits using a diffractive beam splitter. US patent application (application pending) (2021).

\section{ACKNOWLEDGEMENTS}

We acknowledge funding from the Intelligence Advanced Research Projects Activity (IARPA) under contract W911NF-16-1-0114, for the multi-qubit test vehicle and frequency-vs-resistance correlation studies. We thank N. Bronn, M. Carroll, C. Chamberland, A. Cross, J. Gambetta, J. Ku, M. Malekakhlagh, D. McKay, B. Plourde, E. Pritchett, A. Rosenbluth, M. Takita, J. Timmerwilke, and G. Zhu for helpful discussions. We thank E. Porter for coding assistance, Y. Martin and R. Haight for assistance in constructing the laser optics, and R. Patel for photomicroscopy.

\section{AUTHOR CONTRIBUTIONS}

J.B.H. developed the Monte Carlo model with input from J.A.S. and along with J.S.O. analyzed its results. E.M. developed theory to define frequency collisions. J.B.H., J.S.O. and J.M.C. conceived the experiment. V.P.A. and J.B.H. designed the device, and V.P.A. and M.B. fabricated it. M.S. developed apparatus and procedures used for qubit measurement. J.B.H. and M.S. measured the qubit frequencies and J.B.H. analyzed the data. J.-B.Y. and E.J.Z. measured and analyzed room-temperature resistance data. J.S. O., S.R., and E.J.Z. conceived and developed the LASIQ tuning method. J.S.O. and E.J.Z. built the LASIQ apparatus. E.J.Z. undertook the tuning experiments. J.B.H, J.S.O., and E.J.Z. prepared the manuscript, with input from all authors.

\section{COMPETING INTERESTS}

The authors declare no competing interests.

\section{ADDITIONAL INFORMATION}

Supplementary information The online version contains supplementary material available at https://doi.org/10.1038/s41534-021-00464-5.

Correspondence and requests for materials should be addressed to J.B.H. 
Reprints and permission information is available at http://www.nature.com/ reprints

Publisher's note Springer Nature remains neutral with regard to jurisdictional claims in published maps and institutional affiliations.

cc) Open Access This article is licensed under a Creative Commons Attribution 4.0 International License, which permits use, sharing, adaptation, distribution and reproduction in any medium or format, as long as you give appropriate credit to the original author(s) and the source, provide a link to the Creative Commons license, and indicate if changes were made. The images or other third party material in this article are included in the article's Creative Commons license, unless indicated otherwise in a credit line to the material. If material is not included in the article's Creative Commons license and your intended use is not permitted by statutory regulation or exceeds the permitted use, you will need to obtain permission directly from the copyright holder. To view a copy of this license, visit http://creativecommons. org/licenses/by/4.0/.

(c) The Author(s) 2021 